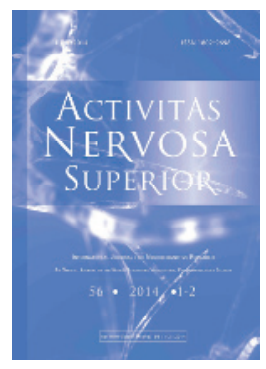

ANS: Journal for Neurocognitive Research Homepage:

WWW.activitas.org

SHORT COMMUNICATION

\title{
AUDITORY ERPS IN CHILDREN WITH DEVELOPMENTAL COORDINATION DISORDER
}

\author{
Irena Holeckova, ${ }^{1}{ }^{*}$ Ladislav Cepicka, ${ }^{2}$ Pavel Mautner, ${ }^{3}$ David Stepanek, ${ }^{1}$ Roman Moucek $^{3}$ \\ ${ }^{1}$ Department of Neurosurgery, Faculty of Medicine and University Hospital in Pilsen, Charles University in \\ Prague, Pilsen, Czech Republic \\ 2Department of Physical Education, Faculty of Education and 3Department of Computer Science and Engineering, \\ Faculty of Applied Sciences, University of West Bohemia in Pilsen, Pilsen, Czech Republic
}

\begin{abstract}
The present study aims to investigate and compare the auditory attention performance of children with developmental coordination disorder (DCD) and normally developing children (NDC) using cognitive evoked potentials (ERPs) in passive conditions. ERPs data showed that children with DCD have less ability to detect small physical differences between acoustic stimuli (no MMN response in DCD children) and have a reduced attentional engagement and stimulus evaluation of salient stimuli (a reduction of P3 amplitude in DCD children). The results of our study suggest that children with DCD do not only suffer from a visuospatial attention deficit as previous studies reported but also have auditory attention deficit.
\end{abstract}

Key words: Developmental coordination disorder; Attention; Auditory ERPs; Children

\section{INTRODUCTION}

Developmental Coordination Disorder (DCD) is a developmental disorder of coordination that is not caused by neurological, psychiatric or physical disorder (APA, 1994, 2000). DCD is manifested by marked deficiencies in the development of physical coordination skills. Children with DCD are defined as children whose motor skills are below average for the given age and intelligence.

The degree of motor development is usually assessed through clinical tests (such as Movement Assessment Battery for Children, Motor Assessment Outcomes Model, Bruininks Oseretsky Test of Motor Proficiency, or Test of Gross Motor Development). Coordination disorders affect both the basic motor skills and the fine motor skills (Polatajko \& Cantin, 2005). Basic motor skills disorders are more important in terms of overall motor performance.

DCD occurs in $6 \%$ of school-age children and significantly affects school performance and reduces the normal daily activities of children. Neuropsychological studies are trying to determine whether DCD is an isolated motor disorder or a combined motor and cognitive disorder, or whether it is only a cognitive disorder. Children with impaired motor skills often have attention deficit disorder and vice versa (Kaplan et al., 1998; Pitche, Piek, \& Barrett; 2002).

${ }^{*}$ Correspondence to: Irena Holeckova, email: holeckova@fnplzen.cz

Received October 18, 2013; accepted July 12, 2014; Act Nerv Super 56(1-2), 37-44; ISSN-1802-9698 
In addition to neuropsychological and behavioral techniques, many neuroimaging methods are available for the study of normal development and developmental neurological, psychological and psychiatric disorders, including electroencephalography (EEG) and evoked potentials (EPs), specifically event-related evoked potentials or cognitive evoked potentials (ERPs) (Taylor, \& Pourcelot, 1995). ERPs represent electrical activity of the brain induced by a stimulus or event and are studied using the odd ball protocol (a sequence of continuous, frequent $=$ standard stimuli that is interrupted by deviant $=$ less frequent stimulus of different physical characteristics). ERPs are used in the study of auditory and visual sensory processes, attention, working memory and other cognitive processes such as speech recognition, evaluation and memory, and others. ERPs have excellent time resolution capacity in the order of milliseconds (ms). Individual components of the ERPs are defined according to their amplitude, polarity and latency after the stimulus. In cognitive neuroscience, the most studied components are those which respond to a less frequent deviant stimulus. These include MMN - mismatch negativity, which represents an automatic orientation response. MMN is also induced under passive conditions, i.e. when the subject's attention is not focused on the stimulus and does not require his/her active cooperation (Naatanen, Gaillard, \& Mantysalo, 1978).

Another frequently used ERP component is P3 wave, which represents more complex cognitive processes, such as focus of attention, stimulus evaluation and stimulus memory. P3 wave is divided into two sub-components: P3a wave and P3b wave. The ability to recall the individual subcomponents is determined by the character of the stimuli and investigation protocol. Response to a deviant stimulus in the active protocol is represented by the P3b wave, registered mainly in the parietal areas. Deviant stimulus, which has specific characteristics: it is salient, significantly different, unexpected and denoted as "novel". This novel stimulus evokes the P3a wave localized in the fronto-central areas, which is recalled under both active and passive conditions (Yago et al., 2003; Holeckova et al., 2006).

It has been demonstrated that children with DCD have a limited ability to distinguish size, angles, area, and shape compared to children with normal development. Meta-analytic study (Wilson \& Mc Kenzie, 1998) revealed a disorder of visuospatial information processing as a major deficit occurring in DCD. Disorders of visuospatial information processing are usually studied using the Posner paradigm, which represents the active protocol investigating the visuospatial attention and orientation, with registration of the P3 component of the ERP (Perchet et al., 2001; Tsai, Wilson, \& Wu, 2008; Tsai et al., 2009).

Approximately $50 \%$ of DCD cases occur together with ADHD (Attention Deficit Hyperactivity Disorder). The high comorbidity between ADHD and DCD supports the idea of a common developmental anomaly of both these disorders (Loh, Piek, \& Barrett, 2011). Studies of ERP using visual and auditory stimuli (Winsberg et al., 1993; Kemner et al., 1996) confirmed an attention deficit for both types of stimuli in children with ADHD not only under the conditions of the active protocol, but also under passive conditions. Thus, it can be assumed, given the expected common anomaly in ADHD and DCD, that children with DCD will have not only visuospatial attention deficit but also an auditory attention disorder.

In our study, we first defined children with DCD based on the results of clinical tests. In the study we used the Test of Gross Motor Development - 2 (TGMD-2), which has the highest validity among the tests used in the diagnosis of gross motor disorders. This test belongs to the most commonly used tools for identification of children with delayed motor development and coordination disorders and is primarily used to identify children who significantly lag behind their peers in the development of basic motor skills (Ulrich, 2000). The test examines simple motor activities such as throwing a ball, walking along a line, etc. Gross score can be transformed into standard scores, percentiles or Gross Motor Quotient (GMQ). DCD is present when the score reaches 89 or less GMQ points.

Main objectives of the study were to assess and compare responses to auditory stimuli measured by event-related evoked potentials at pre-attentive and attentive levels under passive conditions between children with DCD and children with normal motor development 
(NDC) and to test whether children with DCD have only visuospatial attention deficit or also auditory attention deficit.

\section{METHODS}

\subsection{Sample of children}

A total of 16 children aged from 6 to 7 years were enrolled in the study. Mental and neurological disorders were ruled out, and all children had normal hearing.

\subsection{Testing motor skills}

Children were divided into 2 groups based on the results of the Test of Gross Motor Development-2 (TGMD-2). The gross score was transformed into Gross Motor Quotient (GMQ). DCD is present when the score is 89 and less. Based on the results, we assigned 9 children with DCD into group 1 based on the GMQ results with GMQ score of 89 and less. Group 2 included 7 children with NDC, with GMQ score more than 91 points.

\subsection{ERP examination}

The children were examined in a soundproof room, while sitting in a comfortable chair. All the children were instructed to ignore the sound stimuli and closely watch the silent and quiet nature film. Children were asked to limit their volitional movements and eye blinking during registration. The study was approved by the Ethics Committee of the West Bohemian University in Pilsen and parents of these children signed the informed consent form.

\subsection{Stimulation}

Both groups of children were examined using a passive auditory odd ball protocol, in which the children were exposed to three types of auditory stimuli in a randomized order. The stimuli were applied binaurally via headphones. The stimulus intensity was $65 \mathrm{~dB}$ HL. In total, three series of stimuli were applied, each series containing 500 stimuli. Of these, $82 \%$ were standard stimuli (S) - burst tone with duration $75 \mathrm{~ms}$, frequency $800 \mathrm{~Hz}, 14 \%$ were deviant stimuli (D) - burst tone with duration $35 \mathrm{~ms}$, frequency of $800 \mathrm{~Hz}$, and $4 \%$ were rare stimuli = novel stimuli, in our case we used a vocal nonverbal stimulus - child's cry (Snd) with a duration of $600 \mathrm{~ms}$. Each deviant and novel stimulus was followed by at least two standard stimuli. The Stimulus Onset Asynchrony (SOA) was 800 ms.

\subsection{EEG registration}

EEG signal was registered using an EEG cap with 24 electrodes positioned on the head using the 10-20 system. The reference electrode was placed on the forehead, while the grounding electrode was placed on the shoulder. Electrode resistance was kept below $5 \mathrm{k} \Omega$ with $560 \mathrm{~Hz}$ sampling and digital online filtering was $0.5-30 \mathrm{~Hz}$. The EEG was registered on Brain Vision Recorder.

\subsection{Evaluation of EEG and ERP}

EEG signals were analyzed off-line using Brain Vision Analyzer Software. Epochs containing artifacts greater than $100 \mu \mathrm{V}$ (muscle artifacts, ECG, EEG alpha activity) were automatically excluded from further processing. Baseline correction was performed with the prestimulus interval of $50 \mathrm{~ms}$. The EEG signal for S and D stimuli was analyzed with the poststimulus interval of $500 \mathrm{~ms}$ and prestimulus interval $100 \mathrm{~ms}$. For the Snd stimulus, the signal was 
analyzed in the time window of $1300 \mathrm{~ms}$ after the stimulus with prestimulus interval of 100 ms. The component, which had a local maximum within the interval usual for the normal component, was identified as ERP response. We registered N1a and N1c response for the S and D stimuli. We have also registered MMN activity as the difference between D and S stimulus. For the Snd stimulus we registered P3 response. Latency (ms) and amplitude $(\mu \mathrm{V})$ of individual responses was measured. Responses to the respective stimuli were averaged for each individual, and thereafter overall averages of all children in each of the two groups were calculated. Scalp potential maps were generated using the "spherical surface spline interpolation algorithm" (Perrin et al., 1989).

\subsection{Data analysis}

Based on GMQ, children were divided into two divergent groups. We tested a difference in the ERP evaluation between the groups and used one-way analysis of variance, with GMQ being considered factor $A$. To verify the assumption that the variance of all random deviations is the same, we analyzed the one-way analysis of variance using Levene's test.

\section{RESULTS}

\subsection{Response to S stimulus: Negative N1a and N1c wave}

As a response to the standard frequent stimulus, we registered a negative component, known as N1 wave. In our study, this component comprised a two peak wave. The individual peaks were denoted N1a and N1c. The N1a component peaked in the time window between 130 and 140 ms, while the N1c component peaked between 190 and 203 ms (Figure 1). Typical topographical distribution of both waves was recorded on the potential maps, with maximum amplitude in the temporo-parietal areas (Figure 2). Neither the amplitude nor the latency of the N1a and N1c waves showed any statistically significant difference between the two groups (Table 1).

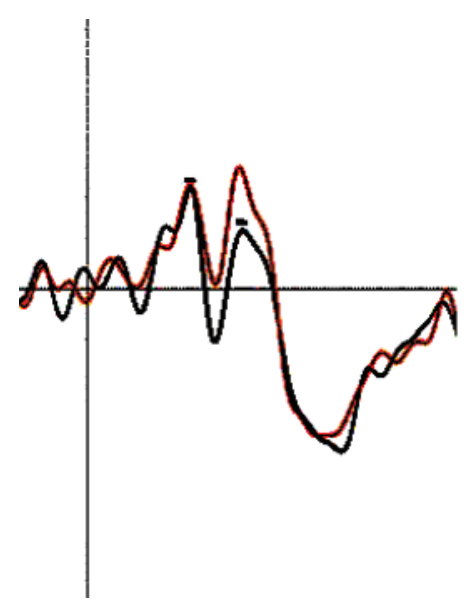

Figure 1. Grand average waveforms N1a and N1c recorded at electrode $\mathrm{T} 4$ for NDC children (red curve) and for DCD children (black curve).
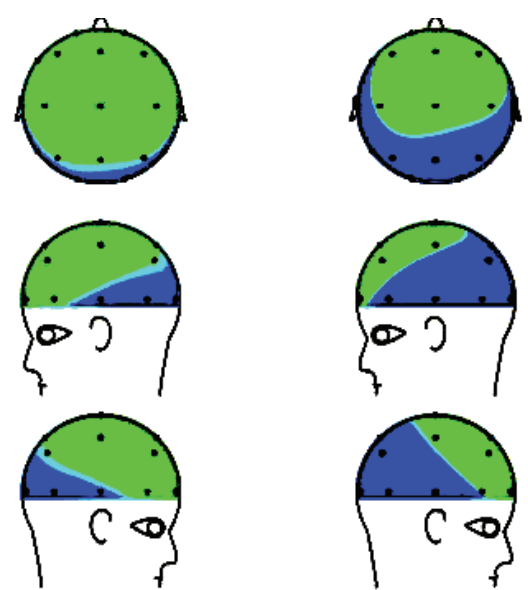

Figure 2. Mean scalp potential distribution of the N1c response in NDC children (blue area). 
Table 1. The latency and amplitude of N1a, N1c, P3a, P3b, N1c responses in DCD (group 1) and NDC children.

\begin{tabular}{|c|c|c|c|c|}
\hline Component & Site & Lat./Ampl. & Group 1 & Group 2 \\
\hline \multirow{4}{*}{$\mathbf{N} 1 \mathbf{a}$} & \multirow{2}{*}{ T3 } & Lat. & $135 \pm 9.8$ & $137 \pm 7.6$ \\
\hline & & Amp. & $-3.3 \pm 1.6$ & $-2.99 \pm 1.4$ \\
\hline & \multirow{2}{*}{ T4 } & Lat. & $137 \pm 7.9$ & $138 \pm 7.6$ \\
\hline & & Amp. & $-2.19 \pm 1.8$ & $-2.12 \pm 1.5$ \\
\hline \multirow{4}{*}{ N1c } & \multirow{2}{*}{ T3 } & Lat. & $192 \pm 19.4$ & $202 \pm 26$ \\
\hline & & Amp. & $4.18 \pm 1.8$ & $-2.8 \pm 2.2$ \\
\hline & \multirow{2}{*}{ T4 } & Lat. & $199 \pm 20 . .3$ & $203 \pm 27$ \\
\hline & & Amp. & $-3.47 \pm 1.7$ & $-2.6 \pm 2.2$ \\
\hline \multirow{2}{*}{ First stage of P3 } & \multirow{2}{*}{$\mathrm{Cz}$} & Lat. & $637 \pm 89$ & $670 \pm 99$ \\
\hline & & Amp. & $10.9 \pm 5.6$ & $11.3 \pm 6.4$ \\
\hline \multirow{2}{*}{ Second stage of P3 } & \multirow{2}{*}{$\mathrm{Pz}$} & Lat. & $844 \pm 78$ & $814 \pm 88$ \\
\hline & & Amp. & $16.79 \pm 11$ & $27.48 \pm 7.1$ \\
\hline
\end{tabular}

3.2. The difference between the responses to stimulus D and S: Mismatch negativity (MMN response)

MMN response was obtained as a numerical difference between the response to stimulus $S$ (frequent stimulus) and response to stimulus D (deviant). This response was recalled only in the group 2 of NDC children and was not present in the group of DCD children. MMN response peaked between 150-170 ms and reached maximum amplitude of $0.5 \mu \mathrm{V}$ in the frontal locations (Figure 3).

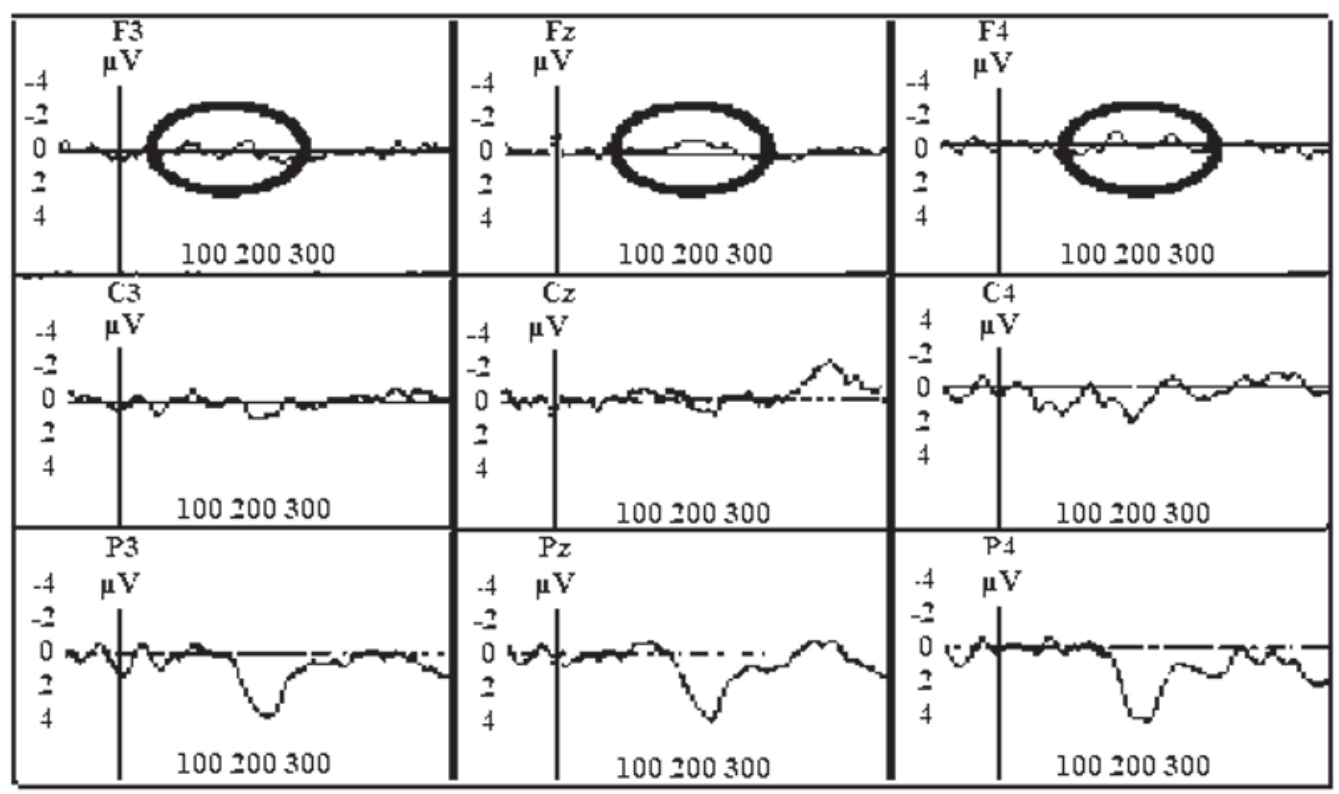

Figure 3. MMN response at F3, Fz and F4 electrodes in NDC children..

\subsection{The response to a complex nonverbal stimulus - child's cry (Snd): P3 wave}

Child's crying evoked a massive P3 response, which was composed of early P3a peak (latency between 635-670 ms) and late peak P3b (latency 814-844 ms) (Table 1). The P3a 
component had its maximum amplitude in the central region $(\mathrm{C} 3, \mathrm{Cz}, \mathrm{C} 4)$, while the $\mathrm{P} 3 \mathrm{~b}$ component had maximum amplitude in the parietal region $(\mathrm{P} 3, \mathrm{Pz}, \mathrm{P} 4)$. No significant differences were recorded between the two groups in the amplitude and latency of the P3a component. For the $\mathrm{P} 3 \mathrm{~b}$ component, we demonstrated significantly lower amplitude in the parietal region $(\mathrm{P} 3, \mathrm{Pz}, \mathrm{P} 4)$ in group 1 of $\mathrm{DCD}$ children $(\mathrm{Pz}: \mathrm{p}=0.036, \mathrm{P} 3$ : $\mathrm{p}=0.014, \mathrm{P} 4: \mathrm{p}=$ 0.038 ) (Figure 4), while no significant difference between the groups was found in the latency of the response.
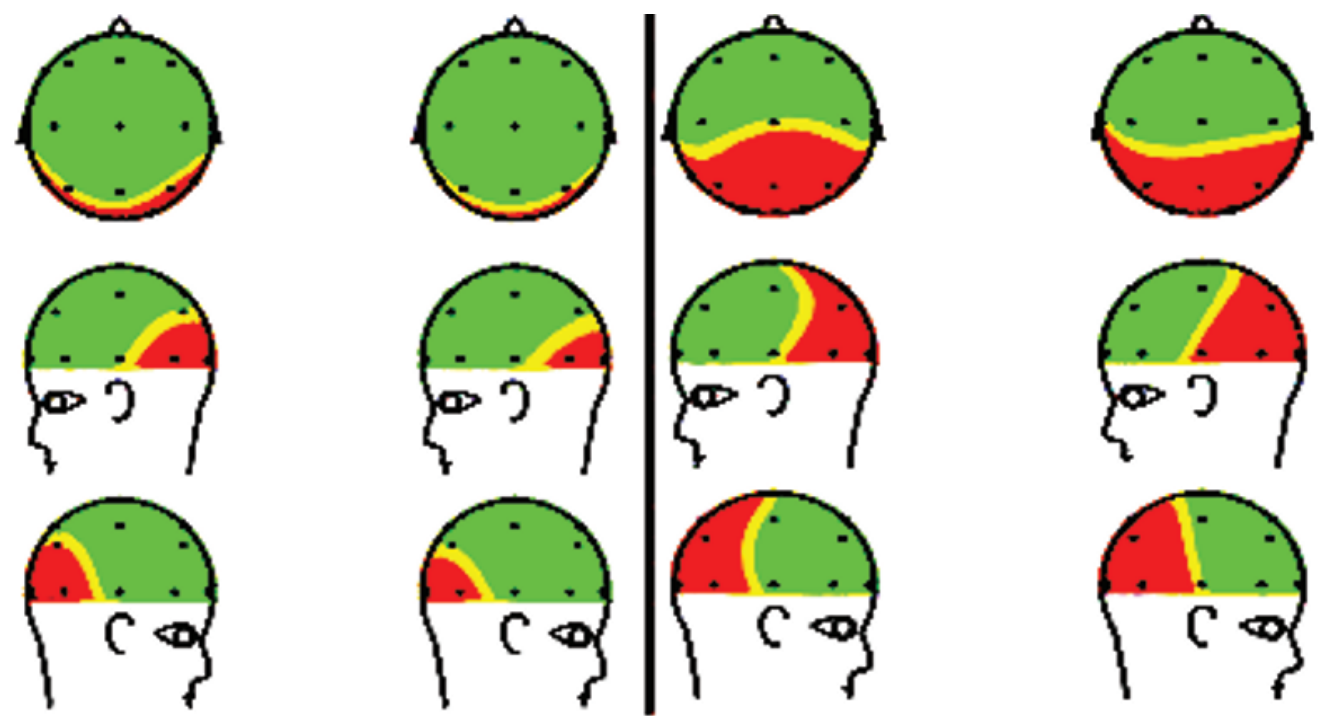

Figure 4. Mean scalp potentials distributions of the P3b response in DCD (the two left columns) and NDC children (the two rights columns).

\section{DISCUSSION}

The objective of our study was to examine whether there are any differences in the evaluation of auditory stimuli of different characteristics under passive conditions, i.e., without focusing on these stimuli in children with DCD compared to the NDC children.

4.1. N1a and N1c response (auditory sensory response): In our study, we demonstrated typical morphology of the auditory sensory response to standard frequent stimulus, the wave N1. This wave consisted of components N1a and N1c with temporo-parietal localization, which is typical for the given age of children. From 8 years of age, this component shows morphology identical to that of the adult population, which means a single peak with a wave of frontal localization with the latency between 80-200 ms (Ponton et al., 2000). In our study, we did not notice any difference in the N1 component between the group of children with DCD and NDC group of children. The result demonstrates no difference between the two groups in perceptual sensory evaluation of auditory stimuli. We also confirmed that this evaluation is different in the given age group compared to the adult population.

4.2. MMN response (preattentive orientation response): MMN response is the electrophysiological correlate of detection and differentiation of physical differences between two stimuli (Naatanen, Gaillard, \& Mantysalo, 1978). MMN is a preattentive, automatic attention switching to different (deviant) stimulus, which occurs in many consecutive series of identical stimuli (standard) (Naatanen \& Michie, 1979). It results from mathematical subtraction of the evoked response elicited by the standard stimulus from the evoked responses elicited by less frequent, deviant stimulus. The processes involved in the 
recall of this wave are automatic, because this wave is also detected under passive conditions, i.e. when diverting one's attention from the stimulus. Its recall requires a neuronal trace preserved in the memory of the standard stimulus and thus, MMN reflects detection of a stimulus with different physical characteristics. It has been reported that MMN is an electrophysiological representative of the working memory. According to some studies, MMN is recalled in children with the same latency and amplitude as in adults (Ceponiene, Cheour \& Naatanen, 1998). Other studies have shown, however, that it may have a longer latency and lower amplitude than in adults (Kurtzberg et al., 1995). In our study, absent recall of MMN in the group of children with DCD suggests that these children have a disorder of automatic detection of physical differences between the two auditory stimuli. Absence of the MMN response in DCD children also suggests a deficit of the working memory.

4.3. $P 3$ component: In our protocol a rare $=$ novel stimulus evoked a P3 answer, where earlier P3a phase typically localized in the fronto-central region, was followed by a late P3b response. This formula of P3 response has been repeatedly reported in responses to a complex stimulus, so each of the phases of the P3 wave represents a different cognitive process (Yago et al., 2003; Holeckova et al., 2006).

In the early P3a phase, we have not recorded any differences between the two groups of children. In contrast, in the P3b phase, we demonstrated lower amplitude of the parietally localized wave in a group of children with DCD. The phase P3a represents a significant attention switching to a salient stimulus. Thus, in our study, the two groups of children show the same degree of attentiveness to a salient stimulus even under passive conditions. It is surprising that a difference between the groups is present in the pre-attentive processing phase (MMN phase). This fact may be explained by a lower difference between the standard and deviant stimuli, which the children with DCD are not able to register and therefore we failed to detect an MMN response in them. However, if a difference between the stimuli is considerable, such as in our case between the standard and novel stimuli, children with DCD are able to register it and pay it the same level of focus and attention shift as children with normal development. The P3b wave is related to processes such as categorization, memory, or assessment of a stimulus in relation to the amount of information contained in the stimulus (Spronk, Jonkman \& Kemner, 2008). Hence, in our study we demonstrated that children with DCD pay less degree of attention to the stimulus and are less committed to its evaluation. We can conclude that as a result, this may affect the attentional control, cognitive flexibility, strategic planning, response preparation or inhibition or organization in time and space.

The results of our study suggest that children with DCD do not only suffer from a visuospatial attention deficit as previous studies reported but also have auditory attention deficit, most probably due to attention deficit in general. They are not able to regulate the amount of attention in situations where is necessary to divide it among multiple stimuli. Along with poor evaluation and storage of information, this could form a basis for coordination disorder, and for learning problems as well.

\section{ACKNOWLEDGEMENT}

This study was supported by grant of Czech Science Agency No. P407/12/1525.

\section{REFERENCES}

American Psychiatric Association (1994). Diagnostic and statistical manual of mental disorders (4th ed). Washington, DC: American Psychiatric Association: 53-55. 
Ceponiene, R., \& Cheour, M., \& Naatanen, R. (1998). Interstimulus interval and auditory event-related potentials in children: evidence for multiple generators. Electroencephalography and Clinical Neurophysiology, 108, 345-54.

Holeckova, I. Fischer, C., Giard, M.H., Delpuech, C., Morlet, D., (2006). Brain responses to a subject's own name uttered by a familiar voice. Brain Research, 1082, 142-52.

Kaplan, B.J., Dewey, D., Crawford, S.G., \& Fisher, G.C. (1998). Deficits in long-term memory are not characteristic of ADHD. Attention Deficit Hyperactivity Disorder. Journal of Clinical and Experimental Neuropsychology, 20, 518-28.

Kemner, C., Verbaten, M.N., Koelega, H.S., Buitelaar, J.K., van der Gaag, R.J., Camfferman, G., \& van Engeland, H. (1996). Event-related brain potentials in children with attention-deficit and hyperactivity disorder: effects of stimulus deviancy and task relevance in the visual and auditory modality. Biological Psychiatry 40, 522-34.

Kurtzberg, D., Vaughan, H.G. Jr, Kreuzer, J.A., \& Fliegler, K.Z. (1995). Developmental studies and clinical application of mismatch negativity: problems and prospects. Ear and Hearing, 16, 105-17.

Loh, P.R., Piek, J.P., \& Barrett, N.C. (2011). Comorbid ADHD and DCD: examining cognitive functions using the WISC-IV. Research in Developmental Disabilities, 32, 1260-9.

Naatanen, R., Gaillard, A.W., \& Mantysalo, S. (1978). Early selective-attention effect on evoked potential reinterpreted. Acta Psychologica (Amst), 42, 313-29.

Naatanen, R., \& Michie, P.T. (1979). Early selective-attention effects on the evoked potential: a critical review and reinterpretation. Biological Psychology, 8, 81-136.

Perchet, C., Revol, O., Fourneret, P., Mauguière, F., \& Garcia-Larrea, L. (2001). Attention shifts and anticipatory mechanisms in hyperactive children: an ERP study using the Posner paradigm. Biol Psychiatry 50(1), 44-57.

Perrin, F., Pernier, J., Bertrand, O., \& Echallier, J.F. (1989). Spherical splines for scalp potential and current density mapping. Electroencephalography and Clinical Neurophysiology, 72, 184-187.

Pitcher, T.M., Piek J.P., \& Barrett, N.C. (2002). Timing and force control in boys with attention deficit hyperactivity disorder: subtype differences and the effect of comorbid developmental coordination disorder. Human Movement Science, 21, 919-45.

Polatajko, H.J., \& Cantin, N. (2005). Developmental coordination disorder (dyspraxia): an overview of the state of the art. Seminars in Pediatric Neurology, 12, 250-8.

Ponton, C.W., Eggermont, J.J., Kwong, B., \& Don, M. (2000). Maturation of human central auditory system activity: evidence from multi-channel evoked potentials. Clinical Neurophysiology, 111, 22036.

Robaey, P. Breton, F., Dugas, M., \& Renault, B. (1992). An event-related potential study of controlled and automatic processes in 6-8-year-old boys with attention deficit hyperactivity disorder. Electroencephalography and Clinical Neurophysiology, 82, 330-40.

Spronk, M., Jonkman L.M., \& Kemner, C. (2008). Response inhibition and attention processing in 5- to 7-year-old children with and without symptoms of ADHD: An ERP study. Clinical Neurophysiology 119, 2738-52.

Taylor, M.J., \& Pourcelot, L. (1995). Cognitive evoked potentials in children: normal and abnormal development. Neurophysiologie Clinique, 25, 130-45.

Tsai, C.L., Pan, C.Y., Cherng. R.J., Hsu, Y.W., \& Chiu, H.H. (2009). Mechanisms of deficit of visuospatial attention shift in children with developmental coordination disorder: a neurophysiological measure of the endogenous Posner paradigm. Brain and Cognition, 71, 246-58.

Tsai, C.L., Wilson P.H., \& Wu, S.K. (2008). Role of visual-perceptual skills (non-motor) in children with developmental coordination disorder. Human Movement Science, 27(4), 649-64.

Ulrich, D.A. (2000). TGMD 2 Test of Gross Motor Development. Austin, TX: Harcourt Education.

Wilson, P.H. \& McKenzie, B.E. (1998) Information Processing Deficits Associated with Developmental Coordination Disorder: A Meta-analysis of Research Findings. Journal of Child Psychology and Psychiatry, 39, 829-840.

Winsberg, B.G., Javitt, D.C., Silipo, G.S., \& Doneshka P. (1993). Mismatch negativity in hyperactive children: effects of methylphenidate. Psychopharmacological Bulletin, 29, 229-33.

Yago, E. et al., (2003). Spatiotemporal dynamics of the auditory novelty-P3 event-related brain potential. Brain Research Cognitive Brain Research, 16, 383-90. 